\title{
GROUPS OF $C^{r, s}$-DIFFEOMORPHISMS RELATED TO A FOLIATION
}

\author{
JACEK LECH and TOMASZ RYBICKI \\ Faculty of Applied Mathematics, AGH University of Science and Technology \\ Al. Mickiewicza 30, 30-059 Kraków, Poland \\ E-mail: lechjace@wms.mat.agh.edu.pl,tomasz@uci.agh.edu.pl
}

\begin{abstract}
The notion of a $C^{r, s}$-diffeomorphism related to a foliation is introduced. A perfectness theorem for the group of $C^{r, s}$-diffeomorphisms is proved. A remark on $C^{n+1}$-diffeomorphisms is given.
\end{abstract}

1. Introduction. The goal of this note is to show that some automorphism groups of a foliated manifold are perfect. Let us recall that a group $G$ is called perfect if $G=[G, G]$, where the commutator subgroup is generated by all commutators $\left[g_{1}, g_{2}\right]=g_{1} g_{2} g_{1}^{-1} g_{2}^{-1}$, $g_{1}, g_{2} \in G$. In terms of homology of groups this means that $H_{1}(G)=G /[G, G]=0$.

The following fundamental result is well-known. Throughout the subscript $c$ indicates the compactly supported subgroup, and the subscript 0 indicates the identity component.

TheOREM 1.1 (Herman, Thurston, Mather). Let $M$ be a smooth manifold, and let $n=$ $\operatorname{dim}(M)$. If $r=1,2, \ldots, \infty, r \neq n+1$, then $\operatorname{Diff}_{c}^{r}(M)_{0}$ is perfect. Consequently, this group is simple as well.

The case $r=\infty$ and $M=T^{n}$ is due to Herman [3] who applied a difficult small denominator theory argument. Next, Thurston [8] used the result of Herman to obtain that $\operatorname{Diff}_{c}^{\infty}(M)_{0}$ is perfect for an arbitrary manifold $M$ (cf. [1] for the proof). By a completely different method Mather [4] proved the first assertion for $r \neq n+1, r$ finite. Finally, the second assertion follows from Epstein [2].

Given a foliated manifold $(M, \mathcal{F})$ a diffeomorphism $f: M \rightarrow M$ is said to be leaf preserving if $f\left(L_{x}\right)=L_{x}$ for all $x \in M$, where $L_{x}$ is the leaf of $\mathcal{F}$ passing through $x$.

2000 Mathematics Subject Classification: 22E65, 57R50.

Key words and phrases: group of $C^{r}$-diffeomorphisms, $C^{r, s}$-diffeomorphism, perfectness, commutator, foliation.

Supported by the AGH grant n. 11.420.04

The paper is in final form and no version of it will be published elsewhere. 
TheOREm 1.2. Let $(M, \mathcal{F})$ be a foliated smooth manifold with $k=\operatorname{dim} \mathcal{F}$, and let $\operatorname{Diff}_{c}^{r}(M, \mathcal{F})$ be the group of leaf preserving diffeomorphisms. Then $\operatorname{Diff}_{c}^{r}(M, \mathcal{F})_{0}$ is perfect, provided $r \leq k$, or $r=\infty$.

The proof of Theorem 1.2 for $r=\infty$ modifies arguments of Herman and Thurston (cf. Rybicki [6]). In the case $r \leq k$ it is easily checked that the proof of Mather [4], II, applies to leaf preserving diffeomorphisms thanks to 'foliated properties' of Mather's operators $P_{i, A}$.

Observe that the group $\operatorname{Diff}_{c}^{r}(M, \mathcal{F})$ is locally contractible (cf. [7]) and, consequently, the identity component of it coincides with the totality of its elements that can be joined to the identity by an isotopy in $\operatorname{Diff}_{c}^{r}(M, \mathcal{F})$.

Our aim is to study here the remaining case of $r>k, r$ finite. In the final section we shall show how a possible analogue of Theorem 1.2 for $r>k+1$ is related to the simplicity of $\operatorname{Diff}_{c}^{n+1}(M)_{0}$. Observe that there exist some strong arguments suggesting that $\operatorname{Diff}_{c}^{n+1}(M)_{0}$ is not simple (Mather [4], III, [5]). This suggests, in turn, that the assertion of Theorem 1.2 for $r>k$ might not be true. However, if we consider groups of leaf preserving diffeomorphisms with some 'loss of smoothness' in the transversal direction then we are able to obtain a positive result.

Given a foliated manifold $(M, \mathcal{F})$ with $k=\operatorname{dim} \mathcal{F}$, let $\operatorname{Diff}^{r, s}(M, \mathcal{F})$ denote the group of leaf preserving $C^{1}$-diffeomorphisms which are of class $C^{r}$ in the tangent direction, and of class $C^{s}$ in the transversal direction, where $1 \leq s \leq r$. (See section 2.)

TheOREM 1.3. The group $\operatorname{Diff}_{c}^{r, s}(M)_{0}$ is perfect, provided $r-s>k+1$.

In the whole paper we exploit the techniques from Mather's fundamental paper [4]. We retain the notation of that paper as far as possible and we recall definitions and facts from it. In particular, the construction of the rolling-up operators is adopted to $C^{r, s}$-diffeomorphisms (section 4 ).

2. Preliminaries. Let $r, s \geq 1$ and $k \geq 1$ be fixed integers. Let $f(x, y)=\left(f_{1}(x, y), y\right)$, where $x \in \mathbb{R}^{k}$ and $y \in \mathbb{R}^{n-k}$.

Definition 2.1. A partial derivative of order $p \geq 1$ of $f$ is called $s$-admissible if it contains at most $s$ derivatives in the direction of the last $n-k$ coordinates. We say that $f$ is of class $C^{r, s}$ if it has all the $s$-admissible partial derivatives up to order $r$ and they are continuous. For $f$ of class $C^{r, s}$ and $1 \leq p \leq r$ we denote by $D^{p, s} f: \mathbb{R}^{n} \rightarrow L^{p}\left(\mathbb{R}^{n}, \mathbb{R}^{n}\right)$ the mapping, called the $p$-th derivative of $f$, consisting of $s$-admissible partial derivatives of $f$ of order $p$, and of zeros in place of partial derivatives of $f$ of order $p$ which are not $s$-admissible. In other words, we may say that $f$ is of class $C^{r, s}$ if $D^{r, s} f$ exists and is continuous. The symbol $C^{r, s}(n, k)$ will stand for the space of all mappings of the form $f(x, y)=\left(f_{1}(x, y), y\right)$, which are of class $C^{r, s}$.

It is clear that $D^{r, s} f=D^{r} f$ if $r \leq s$. In particular we have then the standard derivative formulas for composed mappings

$$
D(f \circ g)=(D f \circ g) \cdot D g
$$


and

$$
\begin{aligned}
D^{r, s}(f \circ g)= & \left(D^{r, s} f \circ g\right) \cdot(D g \times \ldots \times D g)+(D f \circ g) \cdot D^{r, s} g \\
& +\sum C_{i, j_{1}, \ldots, j_{i}}\left(D^{i, s} f \circ g\right) \cdot\left(D^{j_{1}, s} g \times \ldots \times D^{j_{i}, s} g\right),
\end{aligned}
$$

where the sum is over $1<i<r, 1 \leq j_{l}, j_{1}+\ldots+j_{i}=r$ and $C_{i, j_{1}, \ldots, j_{i}}$ are positive integers independent of $f$ and $g$.

Notice that for $r>s$ the above formula (2.2) is no longer valid. The following fact is simple but clue.

Proposition 2.2. Let $f, g \in C^{r, s}(n, k)$. If an entry of the matrix $D^{r, s}(f \circ g)$ on the l.h.s. of (2.2) is an s-admissible partial derivative of $f \circ g$ then the corresponding entry on the r.h.s. is expressed by means of $s$-admissible partial derivatives of orders $\leq r$ of $f$ and $g$.

Proof. It suffices to make the following observation. Any partial derivative in the direction of $x_{i}, i=1, \ldots, k$, of $f \circ g$ cannot produce a partial derivative of $f$ or $g$ in the direction of $y_{j}, j=1, \ldots, n-k$. In case of $g$ this is obvious, in case of $f$ this follows from the fact that $g=\left(g_{1}, g_{2}\right) \in C^{r, s}(n, k)$ satisfies $g_{2}(x, y)=y$ and, consequently, $\frac{\partial g_{2}}{\partial x_{i}}=0$.

DEFINITION 2.3. A modulus of continuity is a continuous, strictly increasing function $\alpha:[0, \infty) \rightarrow \mathbb{R}$, such that $\alpha(0)=0$ and $\alpha(t x) \leq t \alpha(x)$ for every $x \in[0, \infty)$ and $t \geq 1$.

Let $X, Y$ be two metric spaces, and let $\alpha$ be a modulus of continuity. We say that $f: X \rightarrow Y$ is $\alpha$-continuous if there exist $C>0$ and $\varepsilon>0$ such that for every $x_{1}, x_{2} \in X$ and $d_{X}\left(x_{1}, x_{2}\right) \leq \varepsilon$ we have $d_{Y}\left(f\left(x_{1}\right), f\left(x_{2}\right)\right) \leq C \alpha\left(d_{X}\left(x_{1}, x_{2}\right)\right) . f$ is called locally $\alpha$ continuous if each point has a neighborhood $U$ such that $\left.f\right|_{U}$ is $\alpha$-continuous. Obviously these concepts depend on equivalence classes of metrics only.

It is clear that every $f: X \rightarrow Y$ that is Lipschitz, is $\alpha$-continuous for all moduli of continuity $\alpha$. In particular a $C^{1}$-mapping $f: U \rightarrow \mathbb{R}^{n}$, where $U \subset \mathbb{R}^{n}$, is locally $\alpha$-continuous for all moduli of continuity $\alpha$.

The following fact is well-known.

LEMma 2.4. Let $f: X \rightarrow Y$ be a continuous mapping from a compact, convex subset of a normed vector space to a metric space. Then there exists a modulus of continuity $\alpha$ such that $f$ is $\alpha$-continuous.

We say that $f$ is of class $C^{r, s, \alpha}$ if it is $C^{r, s}$ and $D^{r, s} f$ is locally $\alpha$-continuous. Clearly this notion does not depend on the choice of a norm on $L^{r}\left(\mathbb{R}^{n}, \mathbb{R}^{n}\right)$. In the sequel the symbol $C^{r, s,[\alpha]}$ will stand for $C^{r, s}$ or $C^{r, s, \alpha}$. We denote by $\mathcal{D}^{r, s,[\alpha]}(n, k)$ the group of leaf preserving diffeomorphisms of class $C^{r, s,[\alpha]}$ on $\mathbb{R}^{n}$ with compact support which are isotopic to the identity through compactly supported $C^{r, s,[\alpha]}$-isotopies, and by $\mathcal{D}_{K}^{r, s,[\alpha]}(n, k)$ the subgroup of $\mathcal{D}^{r, s,[\alpha]}(n, k)$ of diffeomorphisms supported in $K$.

Proposition 2.5. If $f, g \in C^{r, s,[\alpha]}(n, k)$ then $f \circ g \in C^{r, s,[\alpha]}(n, k)$.

Proof. In fact, this is a consequence of Proposition 2.2.

Proposition 2.6. If $f \in C^{r, s,[\alpha]}(n, k)$ and $f$ has a $C^{1}$-inverse, then $f^{-1} \in C^{r, s,[\alpha]}(n, k)$.

Proof. We have the formula

$$
D\left(f^{-1}\right)=\operatorname{inv} \circ D f \circ f^{-1}
$$


where inv is the inversion in $L\left(\mathbb{R}^{n}, \mathbb{R}^{n}\right)$. It is well-known that inv is of class $C^{\infty} . D\left(f^{-1}\right)$ is of class $C^{r-1, s-1}$. Considering each entry in matrix $D\left(f^{-1}\right)$ it is easy to see that $D^{r, s}\left(f^{-1}\right)$ exists and is continuous.

A leaf preserving mapping of a smooth foliated manifold $f:(M, \mathcal{F}) \rightarrow(M, \mathcal{F})$ is of class $C^{r, s}$ if for every $x \in M$ and for every distinguished chart $(V, v)$ on $(M, \mathcal{F})$ with $f(x) \in V$, there exists a distinguished chart $(U, u)$ on $(M, \mathcal{F})$ with $x \in U, f(U) \subset V$ and $v \circ f \circ u^{-1}$ is $C^{r, s}$.

We define

$$
C^{r, s}(M, \mathcal{F})=\left\{f:(M, \mathcal{F}) \rightarrow(M, \mathcal{F}) \mid f \text { is } C^{r, s} \text { and } f\left(L_{x}\right) \subset L_{x}, \forall x \in M\right\} .
$$

It is obvious that $C^{p+1, s+1}(M, \mathcal{F}) \subset C^{p+1, s}(M, \mathcal{F}) \subset C^{p, s}(M, \mathcal{F})$, for $1 \leq p<r$. By $\operatorname{Diff}^{r, s}(M, \mathcal{F})_{0}$ we denote the group of all leaf preserving $C^{1}$-diffeomorphisms on $M$ of class $C^{r, s}$ which can be joined to the identity by a compactly supported $C^{r, s}$-isotopy.

The following fact can be proved as usual (cf. [1]).

Lemma 2.7. Let $g \in \operatorname{Diff}_{c}^{r, s}(M, \mathcal{F})_{0}$. Then there exist open balls $U_{i}$ and $g_{i} \in \operatorname{Diff}_{c}^{r, s}(M, \mathcal{F})_{0}$, $i=1, \ldots, l$, such that $\operatorname{supp}\left(g_{i}\right) \subset U_{i}$ and $g=g_{1} \ldots g_{l}$.

This fragmentation property enables us to reduce the proof of Theorem 1.3 to the case of $(M, \mathcal{F})=\left(\mathbb{R}^{n}, \mathcal{F}_{k}\right)$, where $\mathcal{F}_{k}=\left\{\mathbb{R}^{k} \times\{\mathrm{pt}\}\right\}$.

As a consequence of Lemmas 2.4 and 2.7 we have the following

LEMMA 2.8. One has

$$
\mathcal{D}^{r, s}(n, k)=\bigcup \mathcal{D}^{r, s, \alpha}(n, k),
$$

where the union is taken over all moduli of continuity $\alpha$.

3. Basic estimates. Let $s \geq 1$ and $0 \leq p \leq r$. For $f \in C^{r, s}\left(\mathbb{R}^{n}, \mathbb{R}^{n}\right)$ we define

$$
\|f\|_{p, s}=\sup _{x \in \mathbb{R}^{n}}\left\|D^{p, s} f(x)\right\| \leq \infty,
$$

and

$$
\|f\|_{p, s, \alpha}=\sup _{x \neq y \in \mathbb{R}^{n}} \frac{\left\|D^{p, s} f(x)-D^{p, s} f(y)\right\|}{\alpha(\|x-y\|)} \leq \infty,
$$

where $\|\cdot\|$ denotes the usual norm in the space of $p$-linear mappings. Further we put $\mu_{p, s}(f)=\|f-\mathrm{id}\|_{p, s}$ and $\mu_{p, s, \alpha}(f)=\|f-\mathrm{id}\|_{p, s, \alpha}$. Moreover, we denote

$$
M_{p, s, \alpha}(f)=\sup \left\{\mu_{1, s, \alpha}(f), \ldots, \mu_{p, s, \alpha}(f)\right\} .
$$

By simple computation we see that $\mu_{1, s}(f) \leq\|f\|_{1, s}+1$ and $\|f\|_{1, s} \leq \mu_{1, s}(f)+1$. Further, we have $\mu_{p, s}(f)=\|f\|_{p, s}$ for $p \geq 2$, and $\mu_{p, s, \alpha}(f)=\|f\|_{p, s, \alpha}$ for $p \geq 1$.

Let $K$ be a closed subset of $\mathbb{R}^{n}$. We define

$$
R_{K}=\sup \left\{\operatorname{dist}\left(q, \overline{\mathbb{R}^{n} \backslash K}\right): q \in \mathbb{R}^{n}\right\} \leq \infty
$$

and

$$
R_{K}^{v}=\sup \left\{\operatorname{dist}\left(q, \overline{\mathbb{R}^{n} \backslash K} \cap L_{q}\right): q \in \mathbb{R}^{n}\right\} \leq \infty .
$$

Here $q \in L_{q} \in \mathcal{F}$. Clearly $R_{K} \leq R_{K}^{v}$.

Proposition 3.1. Let $K$ be a closed interval of $\mathbb{R}^{n}$. 
(1) Assume that $R_{K}<\infty$. Then there exists a constant $C>0$, depending on $R_{K}$ and $\alpha$ such that for all $1 \leq p \leq r$

$$
\mu_{p, s}(f) \leq C \mu_{p, s, \alpha}(f),
$$

whenever $f \in \mathcal{D}_{K}^{r, s, \alpha}(n, k)$.

(2) If $R_{K}^{v}<\infty$ then there exists a constant $C>0$, depending on $R_{K}^{v}$ and $\alpha$ such that for all $1 \leq p<r$

$$
\mu_{p, s, \alpha}(f) \leq C \mu_{p+1, s, \alpha}(f),
$$

for any $f \in \mathcal{D}_{K}^{r, s, \alpha}(n, k)$.

Proof. The inequality in (1) follows by properties of moduli of continuity.

The proof of (2) consists of three steps. First, let us take $\left(x, y^{1}\right),\left(x, y^{2}\right) \in \mathbb{R}^{n}$, where $x \in \mathbb{R}^{k}$ and $y^{1}, y^{2} \in \mathbb{R}^{n-k}$. As $K$ is an interval we can choose $x_{0} \in \mathbb{R}^{k}$ such that $\left(x_{0}, y^{1}\right),\left(x_{0}, y^{2}\right) \in \overline{\mathbb{R}^{n} \backslash K}$ and $\left\|x-x_{0}\right\|=\left\|\left(x, y^{j}\right)-\left(x_{0}, y^{j}\right)\right\| \leq R_{K}^{v}, j=1,2$.

We denote $I_{t}(a, b)=t a+(1-t) b$. Then we have

$$
\begin{array}{rl}
\| D^{p, s} & f\left(x, y^{1}\right)-D^{p, s} f\left(x, y^{2}\right) \| \\
& =\left\|D^{p, s} f\left(x, y^{1}\right)-D^{p, s} f\left(x_{0}, y^{1}\right)+D^{p, s} f\left(x_{0}, y^{2}\right)-D^{p, s} f\left(x, y^{2}\right)\right\| \\
& =\left\|\int_{0}^{1}\left(D^{p+1, s} f\left(I_{t}\left(x, x_{0}\right), y^{1}\right)-D^{p+1, s} f\left(I_{t}\left(x, x_{0}\right), y^{2}\right)\right)\left(x-x_{0}, 0\right) d t\right\| \\
& \leq \sup _{t \in[0,1]}\left\|D^{p+1, s} f\left(I_{t}\left(x, x_{0}\right), y^{1}\right)-D^{p+1, s} f\left(I_{t}\left(x, x_{0}\right), y^{2}\right)\right\|\left\|x-x_{0}\right\| .
\end{array}
$$

Hence

$$
\begin{aligned}
& \frac{\left\|D^{p, s} f\left(x, y^{1}\right)-D^{p, s} f\left(x, y^{2}\right)\right\|}{\alpha\left(\left\|\left(x, y^{1}\right)-\left(x, y^{2}\right)\right\|\right)} \\
& \quad \leq \sup _{z \in \mathbb{R}^{k}} \frac{\left\|D^{p+1, s} f\left(z, y^{1}\right)-D^{p+1, s} f\left(z, y^{2}\right)\right\|}{\alpha\left(\left\|y^{1}-y^{2}\right\|\right)}\left\|x-x_{0}\right\| \\
& \quad \leq R_{K}^{v} \mu_{p+1, s, \alpha}(f) .
\end{aligned}
$$

In the next step we take $\left(x^{1}, y\right),\left(x^{2}, y\right) \in \mathbb{R}^{n}$, where $x^{1}, x^{2} \in \mathbb{R}^{k}$ and $y \in \mathbb{R}^{n-k}$. If $\left\|x^{1}-x^{2}\right\|>1$, we choose $x_{0}^{1}, x_{0}^{2} \in \mathbb{R}^{k}$ such that $\left(x_{0}^{1}, y\right),\left(x_{0}^{2}, y\right) \in \overline{\mathbb{R}^{n} \backslash K},\left\|x^{1}-x_{0}^{1}\right\| \leq R_{K}^{v}$ and $\left\|x^{2}-x_{0}^{2}\right\| \leq R_{K}^{v}$. We obtain

$$
\begin{aligned}
& \frac{\left\|D^{p, s} f\left(x^{1}, y\right)-D^{p, s} f\left(x^{2}, y\right)\right\|}{\alpha\left(\left\|x^{1}-x^{2}\right\|\right)} \\
& \quad \leq \frac{\left\|D^{p, s} f\left(x^{1}, y\right)-D^{p, s} f\left(x_{0}^{1}, y\right)\right\|+\left\|D^{p, s} f\left(x_{0}^{2}, y\right)-D^{p, s} f\left(x^{2}, y\right)\right\|}{\alpha(1)} \\
& \quad \leq \frac{2}{\alpha(1)}\|f\|_{p+1, s}\left(\left\|x^{1}-x_{0}^{1}\right\|+\left\|x^{2}-x_{0}^{2}\right\|\right) .
\end{aligned}
$$

If $\left\|x^{1}-x^{2}\right\| \leq 1$ then

$$
\frac{\left\|D^{p, s} f\left(x^{1}, y\right)-D^{p, s} f\left(x^{2}, y\right)\right\|}{\alpha\left(\left\|x^{1}-x^{2}\right\|\right)} \leq \frac{\|f\|_{p+1, s}\left\|x^{1}-x^{2}\right\|}{\alpha\left(\left\|x^{1}-x^{2}\right\|\right)} \leq \frac{\|f\|_{p+1, s}}{\alpha(1)}
$$

as $\frac{t}{\alpha(t)}$ is an increasing function. In view of (1) one has the inequality in (2). 
Finally, for arbitrary $q, q^{\prime} \in \mathbb{R}^{n}$ we take $q_{0} \in \mathbb{R}^{n}$ with $q-q_{0} \in \mathbb{R}^{k} \times\{0\}$ and $q^{\prime}-q_{0} \in$ $\{0\} \times \mathbb{R}^{n-k}$, and we use the preceding steps of the proof.

Lemma 3.2 ([4]). Let $f$ be a $C^{1}$-diffeomorphism and $\mu_{1, s}(f) \leq \frac{1}{2}$. Then

$$
\mu_{1, s}\left(f^{-1}\right) \leq 2 \mu_{1, s}(f) .
$$

DeFinition 3.3. We say that a polynomial $F$ is admissible if it has no constant term and its coefficients are nonnegative.

Lemma 3.4. Let $1 \leq p \leq r$, let $\alpha$ be a modulus of continuity, and let $K$ be a closed interval of $\mathbb{R}^{n}$ such that $R_{K}^{v}<\infty$.

(1) There exist $\delta_{1}>0$ and $C_{1}>0$ depending on $n, p, \alpha$ and $R_{K}^{v}$ such that

$$
\mu_{p, s, \alpha}(f \circ g) \leq \mu_{p, s, \alpha}(f)+\mu_{p, s, \alpha}(g)+C_{1} \mu_{p, s, \alpha}(f) \mu_{p, s, \alpha}(g)
$$

whenever $f, g \in \mathcal{D}_{K}^{r, s, \alpha}(n, k)$ and $\mu_{p, s, \alpha}(f), \mu_{p, s, \alpha}(g) \leq \delta_{1}$.

(2) For every $\lambda>1$ there exists $\delta_{2}>0$ depending on $n, p, \alpha$ and $R_{K}^{v}$ such that

$$
\mu_{p, s, \alpha}\left(f^{-1}\right) \leq \lambda \mu_{p, s, \alpha}(f)
$$

provided $f \in \mathcal{D}_{K}^{r, s, \alpha}(n, k)$ with $\mu_{p, s, \alpha}(f) \leq \delta_{2}$.

Notice that Lemma 3.4 is formulated only for $K$ such that $R_{K}^{v}<\infty$. But some parts of its proof are valid for the weaker assumption $R_{K}<\infty$. Moreover, the above inequalities are valid for $R_{K}<\infty$, if only $p \leq s$.

Proof. We have for any $q, q^{\prime} \in \mathbb{R}^{n}$

$$
\begin{aligned}
& \frac{\left\|\left(D^{i, s} f \circ g\right)\left(D^{j_{1}, s} g \times \ldots \times D^{j_{i}, s} g\right)(q)-\left(D^{i, s} f \circ g\right)\left(D^{j_{1}, s} g \times \ldots \times D^{j_{i}, s} g\right)\left(q^{\prime}\right)\right\|}{\alpha\left(\left\|q-q^{\prime}\right\|\right)} \\
& \leq \mu_{i, s, \alpha}(f)\left(1+\mu_{1, s}(g)\right)\|g\|_{j_{1}, s} \ldots\|g\|_{j_{i}, s}+2^{i-1}\|f\|_{i, s} \mu_{j_{1}, s, \alpha}(g)\|g\|_{j_{2}, s} \ldots\|g\|_{j_{i}, s} .
\end{aligned}
$$

Then from (2.2), Proposition 2.2 and Proposition 3.1 (1) we have

$$
\begin{aligned}
\mu_{p, s, \alpha}(f \circ g) & \leq \mu_{p, s, \alpha}(f)\left(1+\mu_{1, s}(g)\right)\|g\|_{1, s}^{p}+2^{p-1}\|f\|_{p, s} \mu_{1, s, \alpha}(g)\|g\|_{1, s}^{p-1} \\
& +\mu_{1, s, \alpha}(f)\left(1+\mu_{1, s}(g)\right)\|g\|_{p, s}+\|f\|_{1, s} \mu_{p, s, \alpha}(g) \\
& +\sum C_{i, j_{1}, \ldots, j_{i}}\left(\mu_{i, s, \alpha}(f)\left(1+\mu_{1, s}(g)\right)\|g\|_{j_{1}, s} \ldots\|g\|_{j_{i}, s}\right. \\
& \left.\quad+2^{i-1}\|f\|_{i, s} \mu_{j_{1}, s, \alpha}(g)\|g\|_{j_{2}, s} \ldots\|g\|_{j_{i}, s}\right) \\
& \leq \mu_{p, s, \alpha}(f)+\mu_{p, s, \alpha}(g)+M_{p, s, \alpha}(f) F\left(M_{p, s, \alpha}(g)\right)
\end{aligned}
$$

for arbitrary $f, g \in \mathcal{D}_{K}^{p, s, \alpha}(n, k)$ with $R_{K}<\infty$. Here $F$ is an admissible polynomial independent of $f$ and $g$, and $F=0$ for $p=1$.

Hence, from (3.1) and Proposition 3.1 (2) we obtain (1) for sufficiently small $\mu_{p, s, \alpha}(f)$ and $\mu_{p, s, \alpha}(g)$.

To show (2) we proceed by induction on $p$. First assume that $p=1$ and $R_{K}<\infty$. For $\mu_{1, s}(f)<1$ we have the formula

$$
(D f)^{-1}=\sum_{m=1}^{\infty}(-(D f-\mathrm{Id}))^{m} .
$$


Then from (3.2) we get

$$
\begin{aligned}
\mu_{1, s, \alpha}\left(f^{-1}\right) & \leq \sup _{q \neq q^{\prime}} \frac{\left\|(D f)^{-1}\left(f^{-1}(q)\right)-(D f)^{-1}\left(f^{-1}\left(q^{\prime}\right)\right)\right\|}{\alpha\left(\left\|f^{-1}(q)-f^{-1}\left(q^{\prime}\right)\right\|\right)} \frac{\alpha\left(\left\|f^{-1}\right\|_{1, s}\left\|q-q^{\prime}\right\|\right)}{\alpha\left(\left\|q-q^{\prime}\right\|\right)} \\
& \leq \sqrt{\lambda} \sum_{m=1}^{\infty} \sup \frac{\left\|(D f-\mathrm{Id})^{m}(q)-(D f-\mathrm{Id})^{m}\left(q^{\prime}\right)\right\|}{\alpha\left(\left\|q-q^{\prime}\right\|\right)}
\end{aligned}
$$

since from Proposition 3.1 (1) and Lemma 3.2

$$
\left\|f^{-1}\right\|_{1, s} \leq 1+\mu_{1, s}\left(f^{-1}\right) \leq 1+2 C \mu_{1, s, \alpha}(f) \leq \sqrt{\lambda},
$$

provided $\mu_{1, s, \alpha}(f)$ is small. By using Lemma 3.1 (1) we get

$$
\begin{aligned}
\mu_{1, s, \alpha}\left(f^{-1}\right) & \leq \sqrt{\lambda} \mu_{1, s, \alpha}(f) \sum_{m=1}^{\infty} m \mu_{1, s}(f)^{m-1} \\
& =\sqrt{\lambda} \mu_{1, s, \alpha}(f) \frac{1}{\left(1-\mu_{1, s}(f)\right)^{2}} \leq \lambda \mu_{1, s, \alpha}(f)
\end{aligned}
$$

for sufficiently small $\mu_{1, s, \alpha}(f)$.

For $p \leq s$ we obtain from $(2.2)$

$$
\begin{aligned}
D^{p, s}\left(f^{-1}\right) & =D\left(f^{-1}\right)\left(D^{p, s} f \circ f^{-1}\right)\left(D\left(f^{-1}\right) \times \ldots \times D\left(f^{-1}\right)\right) \\
& +D\left(f^{-1}\right) \sum C_{i, j_{1}, \ldots, j_{i}}\left(D^{i, s} f \circ f^{-1}\right)\left(D^{j_{1}, s}\left(f^{-1}\right) \times \ldots \times D^{j_{i}, s}\left(f^{-1}\right)\right) .
\end{aligned}
$$

Now let $p \geq 2$ and $R_{K}<\infty$. Using (3.3), Propositions 2.2 and 3.1 (1) we have

$$
\begin{aligned}
& \frac{\left\|\left(D\left(f^{-1}\right)\left(D^{p, s} f \circ f^{-1}\right)\left(D\left(f^{-1}\right)\right)^{p}\right)(q)-\left(D\left(f^{-1}\right)\left(D^{p, s} f \circ f^{-1}\right)\left(D\left(f^{-1}\right)\right)^{p}\right)\left(q^{\prime}\right)\right\|}{\alpha\left(\left\|q-q^{\prime}\right\|\right)} \\
& \quad \leq \mu_{1, s, \alpha}\left(f^{-1}\right) \mu_{p, s}(f)\left(1+\mu_{1, s}\left(f^{-1}\right)\right)^{p}+\mu_{p, s, \alpha}(f)\left(1+\mu_{1, s}\left(f^{-1}\right)\right)^{p+2} \\
& \quad+2^{p-1} \mu_{p, s}(f) \mu_{1, s, \alpha}\left(f^{-1}\right)\left(1+\mu_{1, s}\left(f^{-1}\right)\right)^{p} \\
& \quad \leq \mu_{p, s, \alpha}(f)+\mu_{p, s, \alpha}(f) F\left(\mu_{1, s, \alpha}\left(f^{-1}\right)\right),
\end{aligned}
$$

where $F$ is an admissible polynomial.

Similarly, we can estimate the second summand of (3.3) and then

$$
\begin{aligned}
& \frac{\|\left(\left.D\left(f^{-1}\right) \sum C_{i, j_{1}, \ldots, j_{i}}\left(D^{i, s} f \circ f^{-1}\right)\left(D^{j_{1}, s}\left(f^{-1}\right) \times \ldots \times\left(D^{j_{i}, s}\left(f^{-1}\right)\right)\right)\right|_{q^{\prime}} ^{q} \|\right.}{\alpha\left(\left\|q-q^{\prime}\right\|\right)} \\
& \quad \leq \mu_{1, s, \alpha}\left(f^{-1}\right) \sum C_{i, j_{1}, \ldots, j_{i}} \mu_{i, s}(f) \prod_{l=1}^{i}\left(1+\mu_{j_{l}, s}\left(f^{-1}\right)\right) \\
& \quad+\left(1+\mu_{1, s}\left(f^{-1}\right)\right) \sum C_{i, j_{1}, \ldots, j_{i}} \mu_{i, s, \alpha}(f)\left(1+\mu_{1, s}\left(f^{-1}\right)\right) \\
& \quad \cdot \mu_{j_{i_{0}}, s}\left(f^{-1}\right) \prod_{l \neq i_{0}}\left(1+\mu_{j_{l}, s}\left(f^{-1}\right)\right) \\
& \quad+\left(1+\mu_{1, s}\left(f^{-1}\right)\right) \sum C_{i, j_{1}, \ldots, j_{i}} \mu_{i, s}(f) \mu_{j_{1}, s, \alpha}\left(f^{-1}\right) 2^{i-1} \prod_{l=2}^{i}\left(1+\mu_{j_{l}, s}\left(f^{-1}\right)\right) \\
& \leq M_{p-1, s, \alpha}(f) F\left(M_{p-1, s, \alpha}\left(f^{-1}\right)\right) .
\end{aligned}
$$


Now, suppose $R_{K}^{v}<\infty$. From (3.4) and (3.5), by using Proposition 3.1 (2) and induction on $p$, we obtain

$$
\mu_{p, s, \alpha}\left(f^{-1}\right) \leq \mu_{p, s, \alpha}(f)\left(1+F\left(\mu_{p, s, \alpha}(f)\right)\right) \leq \lambda \mu_{p, s, \alpha}(f),
$$

provided $\mu_{p, s, \alpha}(f)$ is sufficiently small.

From the above lemma we obtain by standard arguments the following

Corollary 3.5. Let $K \subset \mathbb{R}^{n}$ be a compact interval, let $p \geq 1$ and let $\alpha$ be a modulus of continuity. Then $(f, g) \mapsto\|f-g\|_{p, s,[\alpha]}$ is a metric on $\mathcal{D}_{K}^{p, s,[\alpha]}(n, k)$. The induced topology is called the $C^{p, s,[\alpha]}$-topology. $\mathcal{D}_{K}^{p, s,[\alpha]}(n, k)$ equipped with the $C^{p, s,[\alpha]}$-topology is a connected topological group.

4. Rolling-up operators $\Psi_{i, A}$. Following Mather [4], I, we let $C_{i}:=\mathbb{R}^{i-1} \times S^{1} \times \mathbb{R}^{n-i}$, where $S^{1} \cong \mathbb{R} / \mathbb{Z}, i=1, \ldots, k$. Let $\pi_{i}: \mathbb{R}^{n} \rightarrow C_{i}$ be the covering projection, and let $\tilde{p}_{i}: \mathbb{R}^{n} \rightarrow \mathbb{R}^{n-1}$ and $p_{i}: C_{i} \rightarrow \mathbb{R}^{n-1}$ be the projections, which omit the $i$-th coordinate. Clearly $p_{i} \circ \pi_{i}=\tilde{p}_{i}$.

The mapping $\pi_{i}: \mathbb{R}^{n} \rightarrow C_{i}$ gives us a system of coordinates in a neighborhood of any point of $C_{i}$, compatible with the foliation $\mathcal{F}_{k, i}=\left\{\mathbb{R}^{i-1} \times S^{1} \times \mathbb{R}^{k-i} \times\{\mathrm{pt}\}\right\}$ on $C_{i}$. Notice that the seminorms introduced above do make also sense on $C_{i}$, and the group $\mathcal{D}^{r, s,[\alpha]}\left(C_{i}, k\right)$ is defined analogously as before.

Let $A \geq 1$ and $K_{i}=[-2,2]^{i} \times[-2 A, 2 A]^{k-i} \times[-2,2]^{n-k}, i=0, \ldots, k$. We have that $K_{0}=[-2 A, 2 A]^{k} \times[-2,2]^{n-k} \supset K_{1} \supset \ldots \supset K_{k}=[-2,2]^{n}$. Next, let $K_{i}^{\prime}=$ $[-2 A, 2 A]^{i-1} \times S^{1} \times[-2 A, 2 A]^{k-i} \times[-2,2]^{n-k}$.

Choose $\tilde{\rho}_{A} \in C^{\infty}(\mathbb{R},[0,1])$ with $\operatorname{supp}\left(\tilde{\rho}_{A}\right)=[-2 A-1,2 A+1]$ and $\tilde{\rho}_{A}=1$ on $[-2 A, 2 A]$. We define $\rho_{A} \in C^{\infty}\left(\mathbb{R}^{n},[0,1]\right)$ by $\rho_{A}(x, y)=\tilde{\rho}_{A}\left(x_{1}\right) \ldots \tilde{\rho}_{A}\left(x_{k}\right)$, where $x=$ $\left(x_{1}, \ldots, x_{k}\right), y=\left(y_{1}, \ldots, y_{n-k}\right)$. Then $\operatorname{supp}\left(\rho_{A}\right)=[-2 A-1,2 A+1]^{k} \times \mathbb{R}^{n-k}$ and $\left.\rho_{A}\right|_{[-2 A, 2 A]^{k} \times \mathbb{R}^{n-k}} \equiv 1$. Let $\tau_{i, A}=\mathrm{Fl}_{1}^{\rho_{A} \partial_{i}} \in \operatorname{Diff}^{\infty}\left(\mathbb{R}^{n}, \mathcal{F}_{k}\right)_{0}$, where $\partial_{i}$ denotes the unit vector field on $\mathbb{R}^{n}$ in the direction of the $i$-th coordinate, and $\mathrm{Fl}_{t}^{X}$ denotes the flow of the vector field $X$. Further we denote by $T_{i}$ the unit translation in the direction of the $i$-th coordinate, i.e. $T_{i}=\mathrm{Fl}_{1}^{\partial_{i}}$.

Let $f \in \mathcal{D}^{r, s, \alpha}(n, k)$ with $\operatorname{supp}(f) \subset K_{0}$ and $\mu_{0, s}(f) \leq \frac{1}{2}$. For $\theta \in C_{i}$ we choose $(x, y) \in$ $\mathbb{R}^{n}$ with $\pi_{i}(x, y)=\theta$ and $x_{i}<-2 A$. Then we choose $N \in \mathbb{N}$ such that $\left(\left(T_{i} f\right)^{N}(x, y)\right)_{i}$ $>2 A$. We define $\Gamma_{i, A}(f): C_{i} \rightarrow C_{i}$ as

$$
\Gamma_{i, A}(f)(\theta)=\pi_{i}\left(\left(T_{i} f\right)^{N}(x, y)\right),
$$

which is independent of the choice of $x$ and $N$.

It is obvious that $\Gamma_{i, A}$ preserves the identity. There exists a neighbourhood $U$ of Id $\in \mathcal{D}^{1, s}(n, k)$ such that

$$
\Gamma_{i, A}: \mathcal{D}_{K_{0}}^{r, s, \alpha}(n, k) \cap U \rightarrow \mathcal{D}_{K_{i}^{\prime}}^{r, s, \alpha}\left(C_{i}, k\right)_{0}
$$

is continuous with respect to the $C^{r, s}$-topology. Moreover we have the following

Lemma 4.1. There exists $\delta>0$ depending on $n, r, \alpha$ and $A$ such that

$$
\mu_{r, s, \alpha}\left(\Gamma_{i, A}(f)\right) \leq 9 A \mu_{r, s, \alpha}(f)
$$


for $f \in \mathcal{D}_{K_{0}}^{r, s, \alpha}(n, k) \cap U$ with $\mu_{r, s, \alpha}(f) \leq \delta$, and

$$
\mu_{r, s, \alpha}\left(\Gamma_{i, A}(f)\right) \leq 9 \mu_{r, s, \alpha}(f)
$$

for $f \in \mathcal{D}_{K_{i}}^{r, s, \alpha}(n, k) \cap U$, where $i>0$, with $\mu_{r, s, \alpha}(f) \leq \delta$.

Proof. Let $N \in \mathbb{N}$ and choose $\varepsilon>0$ such that $\sum_{j=0}^{N-1}(1+\varepsilon)^{j} \leq N+1$. We will show that

$$
\mu_{r, s, \alpha}\left(\left(T_{i} f\right)^{N}\right) \leq\left(1+(1+\varepsilon)+\ldots+(1+\varepsilon)^{N-1}\right) \mu_{r, s, \alpha}(f)
$$

for $\mu_{r, s, \alpha}(f)$ sufficiently small.

By simple computation we have $\mu_{r, s, \alpha}\left(T_{i} f\right)=\mu_{r, s, \alpha}(f)$. Then for $1<m<N$ from (3.1) (which is valid for $R_{K}<\infty$ ), Proposition 3.1 (2), and Lemma 3.4 (1) we obtain arguing by induction

$$
\begin{aligned}
& \mu_{r, s, \alpha}\left(\left(T_{i} f\right)^{m}\right) \\
& \quad \leq \mu_{r, s, \alpha}\left(T_{i} f\right)+\mu_{r, s, \alpha}\left(\left(T_{i} f\right)^{m-1}\right)+M_{r, s, \alpha}\left(T_{i} f\right) F\left(M_{r, s, \alpha}\left(\left(T_{i} f\right)^{m-1}\right)\right) \\
& \quad \leq \mu_{r, s, \alpha}(f)+\left(1+\ldots+(1+\varepsilon)^{m-2}\right) \mu_{r, s, \alpha}(f) \\
& \quad+M_{r, s, \alpha}(f) F\left(\left(1+\ldots+(1+\varepsilon)^{m-2}\right) M_{r, s, \alpha}(f)\right) \\
& \quad \leq \mu_{r, s, \alpha}(f)+\left(1+\ldots+(1+\varepsilon)^{m-2}\right) \mu_{r, s, \alpha}(f) \\
& \quad+\left(R_{K_{0}}^{v}\right)^{r-1} \mu_{r, s, \alpha}(f) F\left(\left(1+\ldots+(1+\varepsilon)^{m-2}\right)\left(R_{K_{0}}^{v}\right)^{r-1} \mu_{r, s, \alpha}(f)\right) \\
& \quad \leq \mu_{r, s, \alpha}(f)+\left(1+\ldots+(1+\varepsilon)^{m-2}\right) \mu_{r, s, \alpha}(f)+\varepsilon\left(1+\ldots+(1+\varepsilon)^{m-2}\right) \mu_{r, s, \alpha}(f) \\
& \quad \leq \mu_{r, s, \alpha}(f)+(1+\varepsilon)\left(1+\ldots+(1+\varepsilon)^{m-2}\right) \mu_{r, s, \alpha}(f) \\
& \quad=\left(1+\ldots+(1+\varepsilon)^{m-1}\right) \mu_{r, s, \alpha}(f)
\end{aligned}
$$

whenever $\mu_{r, s, \alpha}(f)$ is sufficiently small.

Next we choose $N \in \mathbb{N}$ with $8 A+1<N<8 A+3$. Then from (4.1)

$$
\mu_{r, s, \alpha}\left(\Gamma_{i, A}(f)\right)=\mu_{r, s, \alpha}\left(\left(T_{i} f\right)^{N}\right) \leq(N+1) \mu_{r, s, \alpha}(f) \leq 9 A \mu_{r, s, \alpha}(f)
$$

for $f \in \mathcal{D}_{K_{0}}^{r, s, \alpha}(n, k) \cap U$ with $\mu_{r, s, \alpha}(f) \leq \delta$. Analogously

$$
\mu_{r, s, \alpha}\left(\Gamma_{i, A}(f)\right)=\mu_{r, s, \alpha}\left(\left(T_{i} f\right)^{N}\right) \leq \mu_{r, s, \alpha}\left(\left(T_{i} f\right)^{8}\right) \leq 9 \mu_{r, s, \alpha}(f)
$$

for $f \in \mathcal{D}_{K_{i}}^{r, s, \alpha}(n, k) \cap U$ with $\mu_{r, s, \alpha}(f) \leq \delta$, where $i>0$.

Consider the $S^{1}$-action $S^{1} \times C_{i} \rightarrow C_{i}$ given by

$$
\beta \cdot\left(x_{1}, \ldots, \theta, \ldots, x_{k}, y\right)=\left(x_{1}, \ldots, \beta+\theta, \ldots, x_{k}, y\right),
$$

where $y$ stands for $y_{1}, \ldots, y_{n-k}$. Let $G_{i}^{r, s, \alpha}$ denote the group of equivariant $C^{r, s, \alpha}$-diffeomorphisms of $C_{i}$,

$$
G_{i}^{r, s, \alpha}=\left\{f \in \mathcal{D}^{r, s, \alpha}\left(C_{i}, k\right): f(\beta \cdot \theta)=\beta \cdot f(\theta) \forall \beta \in S^{1} \forall \theta \in C_{i}\right\} .
$$

Proposition 4.2. Let $\alpha$ be a modulus of continuity and $A \geq 1$. Then there exists a neighborhood $U_{A}$ of id $\in \operatorname{Diff}_{c}^{1}\left(\mathbb{R}^{n}\right)$ depending on $n, r, \alpha$ and $A$ such that for $f, g \in$ $\mathcal{D}_{K_{0}}^{r, s, \alpha}(n, k) \cap U_{A}$ with $\Gamma_{i, A}(f) \Gamma_{i, A}(g)^{-1} \in G_{i}^{r, s, \alpha}$, the mappings $\tau_{i, A} f$ and $\tau_{i, A} g$ are conjugate in $\mathcal{D}^{r, s, \alpha}(n, k)$.

For the proof, see [4], I.

Now we will follow Mather [4], I, to define rolling-up operators $\Psi_{i, A}$. It is important that all steps of definition $\Psi_{i, A}$ are 'leaf preserving'. 
Let $f \in U_{A} \cap \mathcal{D}_{K_{0}}^{r, s, \alpha}(n, k)$, where $U_{A}$ is as in Proposition 4.2. We can find $g \in C^{1}\left(C_{i}, C_{i}\right)$ such that $g \in G_{i}^{1, s, \alpha}$ and $g=\Gamma_{i, A}(f)$ on $\left\{\theta \in C_{i}: \theta_{i}=0\right\}$ by the formula

$$
g\left(x_{1}, \ldots, \theta_{i}, \ldots, x_{k}, y\right)=\Gamma_{i, A}(f)\left(x_{1}, \ldots, 0, \ldots, x_{k}, y\right)+\left(0, \ldots, \theta_{i}, \ldots, 0\right) .
$$

Moreover $g$ depends continuously on $f$, so we can shrink $U_{A}$ such that $g \in \mathcal{D}_{K_{i}^{\prime}}^{1, s}\left(C_{i}, k\right)_{0}$. It is easily seen that

$$
\mu_{r, s, \alpha}(g) \leq \mu_{r, s, \alpha}\left(\Gamma_{i, A}(f)\right) .
$$

Let $h=g^{-1} \Gamma_{i, A}(f) \in \mathcal{D}_{K_{i}^{\prime}}^{1, s}\left(C_{i}, k\right)_{0}$.

We identify $g, h$ and $\Gamma_{i, A}(f)=g h$ with periodic diffeomorphisms of $\mathbb{R}^{n}$ supported in $K_{i}^{\prime \prime}=[-2 A, 2 A]^{i-1} \times \mathbb{R} \times[-2 A, 2 A]^{k-i} \times[-2,2]^{n-k}$. By abuse these periodic diffeomorphisms will be denoted by the same letters. It is easily seen that the lifted diffeomorphisms have the same norms $\mu_{r, s,[\alpha]}$ as the initial ones.

In order to perform further steps of the construction we need the following analog of Proposition 3.1 for periodic diffeomorphisms.

Proposition 4.3. Let $f$ be a periodic $C^{r, s}$-diffeomorphisms of $\mathbb{R}^{n}$ with period 1 with respect to the variable $x_{i}$ for some $i=1, \ldots, k$, and let $\left.f\right|_{\left\{x \in \mathbb{R}^{n}: x_{i}=0\right\}}=$ Id. Then for every $p=0, \ldots, r$

(1) $\mu_{p, s}(f) \leq \mu_{r, s}(f)$, and

(2) $\mu_{p, s, \alpha}(f) \leq C^{r} \mu_{r, s, \alpha}(f)$, where $C>0$ depends on $\alpha$.

Proof. Note that $f$ is periodic with period 1 if and only if $(f-\mathrm{Id})\left(x_{1}, \ldots, x_{i}+1, \ldots, x_{k}, y\right)$ $=(f-\mathrm{id})(x, y)$. In order to show $(1)$ we just integrate partial derivatives of $f-$ Id with respect to $x_{i}$, and this procedure does not change $s$ in $\mu_{j, s}$. Here we use the periodicity of $f-\operatorname{Id}$ and of its derivatives, and the condition $\left.f\right|_{\left\{x \in \mathbb{R}^{n}: x_{i}=0\right\}}=\operatorname{Id}$ for $p=0$.

Now we prove (2). Let $\beta \in \mathbb{N}^{n},|\beta|=p, \beta=\left(\beta^{\prime}, \beta^{\prime \prime}\right)$, where $\beta^{\prime} \in \mathbb{R}^{k}$ and $\beta^{\prime \prime} \in \mathbb{R}^{n-k}$ and $\left|\beta^{\prime \prime}\right| \leq s$. First, let us take $\left(x, y^{1}\right),\left(x, y^{2}\right) \in \mathbb{R}^{n}$, where $x \in \mathbb{R}^{k}$ and $y^{1}, y^{2} \in \mathbb{R}^{n-k}$. We may write

$$
D^{\beta} f\left(x, y_{j}\right)=\frac{\partial^{\left|\beta^{\prime}\right|}}{\partial x^{\beta^{\prime}}}\left(D^{\beta^{\prime \prime}} f\left(x, y_{j}\right)\right)
$$

and $D^{\beta^{\prime \prime}} f\left(x, y_{j}\right)$ viewed as a function of $x$, is a periodic function with period 1 with respect to $x_{i}$ which is equal to 0 on $\left\{x \in \mathbb{R}^{n}: x_{i}=0\right\}$, for $j=1,2$. Then

$$
\begin{aligned}
\left\|D^{\beta} f\left(x, y_{1}\right)-D^{\beta} f\left(x, y_{2}\right)\right\| & =\left\|\frac{\partial^{\left|\beta^{\prime}\right|}}{\partial x^{\beta^{\prime}}}\left(D^{\beta^{\prime \prime}} f\left(x, y_{1}\right)-D^{\beta^{\prime \prime}} f\left(x, y_{2}\right)\right)\right\| \\
& \leq \sup _{x \in \mathbb{R}^{k}}\left\|\frac{\partial^{\left|\beta_{i}^{\prime}\right|}}{\partial x^{\beta_{i}^{\prime}}}\left(D^{\beta^{\prime \prime}} f\left(x, y_{1}\right)-D^{\beta^{\prime \prime}} f\left(x, y_{2}\right)\right)\right\| \\
& =\sup _{x \in \mathbb{R}^{k}}\left\|D^{\beta_{i}} f\left(x, y_{1}\right)-D^{\beta_{i}} f\left(x, y_{2}\right)\right\| \\
& \leq \sup _{x \in \mathbb{R}^{k}}\left\|D^{p+1, s} f\left(x, y_{1}\right)-D^{p+1, s} f\left(x, y_{2}\right)\right\|,
\end{aligned}
$$

where $\beta_{i}^{\prime}=\left(\beta_{1}, \ldots, \beta_{i}+1, \ldots, \beta_{k}\right)$ and $\beta_{i}=\left(\beta_{i}^{\prime}, \beta^{\prime \prime}\right)$, after integration along the $x_{i}$-axis. 
Therefore

$$
\begin{aligned}
\frac{\left\|D^{p, s} f\left(x, y^{1}\right)-D^{p, s} f\left(x, y^{2}\right)\right\|}{\alpha\left(\left\|\left(x, y^{1}\right)-\left(x, y^{2}\right)\right\|\right)} & \leq \frac{\left\|D^{p+1, s} f\left(x, y^{1}\right)-D^{p+1, s} f\left(x, y^{2}\right)\right\|}{\alpha\left(\left\|y^{1}-y^{2}\right\|\right)} \\
& \leq \mu_{p+1, s, \alpha}(f) .
\end{aligned}
$$

In the second case, where $\left(x^{1}, y\right),\left(x^{2}, y\right) \in \mathbb{R}^{n}$ with $x^{1}, x^{2} \in \mathbb{R}^{k}$ and $y \in \mathbb{R}^{n-k}$, we proceed as in the proof of Proposition 3.1, by using the periodicity of $f$. The general case follows from the first two cases.

We have $\mu_{1, s, \alpha}\left(g^{-1}\right) \leq 2 \mu_{1, s, \alpha}(g)$. From (3.4), (3.5) (bearing in mind that $R_{K_{i}^{\prime \prime}}=$ $2 A<\infty)$, the above inequalities and Propositions 3.1 (1) and 4.3 we have by induction on $p$

$$
\begin{aligned}
\mu_{p, s, \alpha}\left(g^{-1}\right) & \leq \mu_{p, s, \alpha}(g)+M_{p, s, \alpha}(g) F\left(M_{p-1, s, \alpha}\left(g^{-1}\right)\right) \\
& \leq \mu_{p, s, \alpha}\left(\Gamma_{i, A}(f)\right)+M_{p, s, \alpha}\left(\Gamma_{i, A}(f)\right) F\left(M_{p-1, s, \alpha}\left(\Gamma_{i, A}(f)\right)\right) \\
& \leq 9 A \mu_{p, s, \alpha}(f)+C^{p} 9 A \mu_{p, s, \alpha}(f) F\left(C^{p-1} 9 A \mu_{p-1, s, \alpha}(f)\right) \\
& \leq C^{p} A \mu_{p, s, \alpha}(f)
\end{aligned}
$$

if $\mu_{p, s, \alpha}(f)$ is sufficiently small. Here $C$ is independent of $A$. Therefore

$$
\begin{aligned}
\mu_{r, s, \alpha}(h) & \leq \mu_{r, s, \alpha}\left(g^{-1}\right)+\mu_{r, s, \alpha}\left(\Gamma_{i, A}(f)\right)+M_{r, s, \alpha}\left(g^{-1}\right) F\left(M_{r, s, \alpha}\left(\Gamma_{i, A}(f)\right)\right) \\
& \leq C^{r} A \mu_{r, s, \alpha}(f)
\end{aligned}
$$

for $f \in U_{A} \cap \mathcal{D}_{K_{0}}^{r, s, \alpha}(n, k)$ with $\mu_{r, s, \alpha}(f) \leq \delta_{1}$.

Fix a function $\xi \in C^{\infty}(\mathbb{R},[0,1])$ of period 1 , which equals 0 near $m$ and equals 1 near $m+\frac{1}{2}$, where $m \in \mathbb{Z}$. We define functions

$$
h_{-}=\left(\xi \circ \mathrm{pr}_{i}\right) \cdot(h-\mathrm{Id})+\mathrm{Id}, \quad h_{+}=h_{-}^{-1} h .
$$

Shrinking $U_{A}$ if necessary, $h_{-}, h_{+} \in \operatorname{Diff}_{K_{i}^{\prime \prime}}^{1, s}\left(\mathbb{R}^{n}\right)_{0}$.

Then we have

$$
\begin{aligned}
\mu_{r, s, \alpha}\left(h_{-}\right) & \leq \sup _{q \neq q^{\prime} \in \mathbb{R}^{n}} \frac{\left\|\left.\left(D^{r, s}\left(\left(\xi \circ \mathrm{pr}_{i}\right)(h-\mathrm{Id})\right)\right)\right|_{q^{\prime}} ^{q}\right\|}{\alpha\left(\left\|q-q^{\prime}\right\|\right)} \\
& \leq \sum_{j=0}^{r}\left(\begin{array}{l}
r \\
j
\end{array}\right)\left[\sup _{q \neq q^{\prime}} \frac{\left\|\left.\left(D^{j, s}\left(\xi \circ \mathrm{pr}_{i}\right)\right)\right|_{q^{\prime}} ^{q}\right\|\left\|D^{r-j, s}(h-\mathrm{Id})(q)\right\|}{\alpha\left(\left\|q-q^{\prime}\right\|\right)}\right. \\
& \left.+\sup _{q \neq q^{\prime}} \frac{\left\|D^{j, s}\left(\xi \circ \mathrm{pr}_{i}\right)\left(q^{\prime}\right)\right\|\left\|\left.\left(D^{r-j, s}(h-\mathrm{Id})\right)\right|_{q^{\prime}} ^{q}\right\|}{\alpha\left(\left\|q-q^{\prime}\right\|\right)}\right] \\
& \leq \sum_{j=0}^{r}\left(\begin{array}{l}
r \\
j
\end{array}\right)\left(\left\|\xi \circ \operatorname{pr}_{i}\right\|_{j, s, \alpha} \mu_{r-j, s}(h)+\left\|\xi \circ \operatorname{pr}_{i}\right\|_{j, s} \mu_{r-j, s, \alpha}(h)\right) \\
& \leq C_{1} \mu_{r, s, \alpha}(h) .
\end{aligned}
$$

By Lemma 3.4 and Proposition 4.3 there exists $C_{2}>0$ independent of $A$ such that

$$
\mu_{r, s, \alpha}\left(h_{+}\right) \leq C_{2} A \mu_{r, s, \alpha}(f)
$$


for small $\mu_{r, s, \alpha}(f)$. Let us take

$$
E_{-}=\left\{(x, y) \in \mathbb{R}^{n}:-1 \leq x_{i} \leq 0\right\}, \quad E_{+}=\left\{(x, y) \in \mathbb{R}^{n}: \frac{1}{2} \leq x_{i} \leq \frac{3}{2}\right\},
$$

and define $\Psi_{i, A}(f)$ by

$$
\left.\Psi_{i, A}(f)\right|_{E_{+}}=\left.h_{+}\right|_{E_{+}},\left.\quad \Psi_{i, A}(f)\right|_{E_{-}}=\left.h_{-}\right|_{E_{-}},
$$

and $\left.\Psi_{i, A}(f)\right|_{\mathbb{R}^{n} \backslash\left(E_{-} \cup E_{+}\right)}=\mathrm{Id}$.

Then we have

$$
\Gamma_{i, A}(f) \Gamma_{i, A}\left(\Psi_{i, A}(f)\right)^{-1}=\Gamma_{i, A}(f) h^{-1}=g \in G_{i}^{r, s, \alpha} .
$$

Shrinking $U_{A}$ if necessary and using Proposition 4.2 we see that $\tau_{i, A} f$ and $\tau_{i, A} \Psi_{i, A}(f)$ are conjugate.

Summing up the above considerations we have the following

Proposition 4.4. There exist a neighborhood $U_{A}$ of $\mathrm{Id} \in \operatorname{Diff}_{K_{0}}^{1}\left(\mathbb{R}^{n}\right)_{0}$ and the operators

$$
\Psi_{i, A}: U_{A} \rightarrow \operatorname{Diff}_{K_{0}}^{1}(n, k), \quad 1 \leq i \leq k,
$$

with the following properties.

(1) $\Psi_{i, A}$ preserves the identity.

(2) $\Psi_{i, A}: U_{A} \cap \mathcal{D}_{K_{i-1}}^{r, s, \alpha}(n, k) \rightarrow \mathcal{D}_{K_{i}}^{r, s, \alpha}(n, k)$ is continuous with respect to the $C^{r, s}$ topology.

(3) For every $f \in U_{A} \cap \mathcal{D}^{r, s, \alpha}(n, k)$ we have

$$
[f]=\left[\Psi_{i, A}(f)\right] \in H_{1}\left(\mathcal{D}^{r, s, \alpha}(n, k)\right) .
$$

(4) There exists $\delta>0$ depending on $n, r, \alpha, A$, and $C>1$ depending on $n, r, \alpha$ but independent of $A$ with

$$
\mu_{r, s, \alpha}\left(\Psi_{i, A}(f)\right) \leq C A \mu_{r, s, \alpha}(f)
$$

for all $f \in U_{A} \cap \mathcal{D}^{r, s, \alpha}(n, k)$ with $\mu_{r, s, \alpha}(f) \leq \delta$.

5. Proof of the Theorem 1.3. Let $f \in \mathcal{D}^{r, s}(n, k)$. In view of Lemmas 2.7 and 2.8 we may assume that $f \in \mathcal{D}_{[-2,2] n}^{r, s, \alpha}(n, k)$. Moreover, $f$ can be chosen sufficiently close to the identity in the $C^{r, s, \alpha}$-topology due to Corollary 3.5. We have to show that $f$ belongs to the commutator subgroup $\left[\mathcal{D}^{r, s, \alpha}(n, k), \mathcal{D}^{r, s, \alpha}(n, k)\right]$.

Let us take $\chi_{A} \in \operatorname{Diff}_{c}^{\infty}\left(\mathbb{R}^{n}\right)_{0}$ such that for any $(x, y) \in[-2,2]^{k} \times \mathbb{R}^{n-k}$ one has $\chi_{A}(x, y)=(A x, y)$. Then for any $g \in \mathcal{D}^{r, s, \alpha}(n, k)$ we define $g_{0}=\chi_{A} f g \chi_{A}^{-1}$, and $g_{i}=$ $\Psi_{i, A}\left(g_{i-1}\right)$ for $i=1, \ldots, k$. It is obvious by Proposition 4.4 that $\left[g_{k}\right]=[f g]$.

Lemma 5.1. Let $r-s>k+1$. Then there exist $A \geq 1$ and $\varepsilon_{0}>0$ such that for every $0<\varepsilon<\varepsilon_{0}$ and $f, g \in \mathcal{D}_{[-2,2]^{n}}^{r, s, \alpha}(n, k)$ with $\mu_{r, s, \alpha}(f), \mu_{r, s, \alpha}(g) \leq \varepsilon$ we have $\mu_{r, s, \alpha}\left(g_{k}\right) \leq \varepsilon$.

Proof. We can choose $A$ so large that $3 C^{k} A^{1-r+s+k} \leq 1$, where $C$ is the constant from Proposition 4.4 (4). There exists $\varepsilon_{0}>0$ such that we have

$$
\mu_{r, s, \alpha}(f g) \leq \mu_{r, s, \alpha}(f)+\mu_{r, s, \alpha}(g)+C_{1} \mu_{r, s, \alpha}(f) \mu_{r, s, \alpha}(g) \leq 3 \varepsilon,
$$


for every $0<\varepsilon \leq \varepsilon_{0}$ and $f, g$ as above. In view of definition of $D^{r, s}$ we have

$$
\left\|D^{r, s}\left(\chi_{A} f g \chi_{A}^{-1}\right)(x, y)\right\| \leq\left\|A^{1-r+s} D^{r, s}(f \circ g)\left(\frac{1}{A} x, y\right)\right\| .
$$

Therefore, for $q=(x, y), q^{\prime}=\left(x^{\prime}, y^{\prime}\right)$

$$
\begin{aligned}
\mu_{r, s, \alpha}\left(\chi_{A} f g \chi_{A}^{-1}\right) & =\sup _{q \neq q^{\prime}} \frac{\left\|D^{r, s}\left(\chi_{A} f g \chi_{A}^{-1}\right)(q)-D^{r, s}\left(\chi_{A} f g \chi_{A}^{-1}\right)\left(q^{\prime}\right)\right\|}{\alpha\left(\left\|q-q^{\prime}\right\|\right)} \\
& \leq A^{1-r+s} \sup _{q \neq q^{\prime}} \frac{\left\|D^{r, s}(f g)\left(\frac{1}{A} x, y\right)-D^{r, s}(f g)\left(\frac{1}{A} x^{\prime}, y^{\prime}\right)\right\|}{\alpha\left(\left\|q-q^{\prime}\right\|\right)} \\
& \leq A^{1-r+s} \sup _{q \neq q^{\prime}} \frac{\left\|D^{r, s}(f g)\left(\frac{1}{A} x, y\right)-D^{r, s}(f g)\left(\frac{1}{A} x^{\prime}, y^{\prime}\right)\right\|}{\alpha\left(\left\|\left(\frac{1}{A} x, y\right)-\left(\frac{1}{A} x^{\prime}, y^{\prime}\right)\right\|\right)} \\
& \leq A^{1-r+s} \mu_{r, s, \alpha}(f g) \leq 3 A^{1-r+s} \varepsilon .
\end{aligned}
$$

If $\varepsilon_{0} \leq \delta$, where $\delta$ is the constant from Proposition 4.4 (4), we obtain

$$
\mu_{r, s, \alpha}\left(g_{k}\right) \leq C^{k} A^{k} \mu_{r, s, \alpha}\left(\chi_{A} f g \chi_{A}^{-1}\right) \leq 3 C^{k} A^{1-r+s+k} \varepsilon \leq \varepsilon .
$$

LEMMA 5.2. Let $a>0$. The set

$$
L=\left\{h \in \mathcal{D}_{[-a, a]^{n}}^{r, s, \alpha}(n, k): \mu_{r, s, \alpha}(h) \leq \varepsilon\right\}
$$

equipped with the $C^{r, s}$-topology has the fixed-point property, i.e. every continuous mapping $L \rightarrow L$ has a fixed point.

Proof. Let us consider

$$
L^{\prime}=\left\{h^{\prime}: h^{\prime}+\operatorname{Id} \in L\right\} \subset\left(C_{[-a, a]^{n}}^{r, s}\left(\mathbb{R}^{n}, \mathbb{R}^{n}\right),\|\cdot\|_{r, s}\right) .
$$

Here $h^{\prime}(x, y)=\left(h_{1}^{\prime}(x, y), 0\right)$ is of class $C^{r, s, \alpha}$.

We have the homeomorphism $L \ni h \mapsto h-\mathrm{Id} \in L^{\prime} . L^{\prime}$ is closed in $\left(C_{[-a, a]^{n}}^{r, s}\left(\mathbb{R}^{n}, \mathbb{R}^{n}\right)\right.$, $\left.\|\cdot\|_{r, s}\right)$. Let us take

$$
T:\left(C_{[-a, a]^{n}}^{r, s}\left(\mathbb{R}^{n}, \mathbb{R}^{n}\right),\|\cdot\|_{r, s}\right) \ni h \mapsto D^{r, s} h \in\left(C_{[-a, a]^{n}}^{0}\left(\mathbb{R}^{n}, L^{r}\left(\mathbb{R}^{n}, \mathbb{R}^{n}\right)\right),\|\cdot\|_{\mathrm{sup}}\right) .
$$

$T$ is continuous as

$$
\|T h\|_{\text {sup }}=\sup _{x \in \mathbb{R}^{n}}\left\|D^{r, s} h(x)\right\|=\|h\|_{r, s} .
$$

For every $h \in L^{\prime}$ we have

$$
\begin{gathered}
\|T h(x)-T h(y)\|=\left\|D^{r, s} h(x)-D^{r, s} h(y)\right\| \\
\leq \frac{\left\|D^{r, s} h(x)-D^{r, s} h(y)\right\|}{\alpha(\|x-y\|)} \alpha(\|x-y\|) \leq \varepsilon \alpha(\|x-y\|),
\end{gathered}
$$

so $T\left(L^{\prime}\right)$ is equicontinuous, and it is bounded in view of (5.1). By Ascoli-Arzela's theorem, the set $T\left(L^{\prime}\right)$ is relative compact in $\left(C_{[-a, a]^{n}}^{0}\left(\mathbb{R}^{n}, L^{r}\left(\mathbb{R}^{n}, \mathbb{R}^{n}\right)\right),\|\cdot\|_{\text {sup }}\right)$, so it is compact.

Hence $L^{\prime}$ and $L$ are compact. Since $L$ is a convex subset of a Fréchet space, by Schauder-Tychonoff's theorem every continuous map $L \rightarrow L$ has a fixed point.

We choose $\varepsilon>0$ as in Lemma 5.1. Then $L$ has the fixed-point property, and the mapping

$$
\mathcal{D}_{[-2,2]^{n}}^{r, s, \alpha}(n, k) \ni g \mapsto g_{k} \in \mathcal{D}_{[-2,2]^{n}}^{r, s, \alpha}(n, k),
$$


is continuous with respect to the $C^{r, s}$-topology. Hence there exists $g \in L$ such that $g=g_{k}$. Therefore

$$
[f][g]=[f g]=\left[g_{k}\right]=[g] \in H_{1}\left(\mathcal{D}^{r, s, \alpha}(n, k)\right) .
$$

and $[f]=[\mathrm{Id}] \in H_{1}\left(\mathcal{D}^{r, s, \alpha}(n, k)\right)$. This completes the proof.

6. Remark on $C^{n+1}$-diffeomorphisms. It is still not known whether the group $\operatorname{Diff}_{c}^{n+1}(M)_{0}$ is perfect and simple. Mather in [5] considered the geometric transfer map and proved that linearized forms of commutator equations are true iff $r \neq n+1$. This result strongly suggests that $\operatorname{Diff}_{c}^{n+1}(M)_{0}$ is not perfect.

Observe that the perfectness of $\operatorname{Diff}_{c}^{n+1}(M)_{0}$ is strictly related to the perfectness of $\operatorname{Diff}_{c}^{r}(M, \mathcal{F})_{0}$ for large $r$. In fact, let $f \in \operatorname{Diff}_{c}^{n+1}(M)_{0}$ be sufficiently close to Id, and let $0<k<n$. In view of Lemma 2.7 we may assume that $f \in \operatorname{Diff}_{c}^{n+1}\left(\mathbb{R}^{n}\right)_{0}$. Then there exist $g \in \operatorname{Diff}_{c}^{n+1}\left(\mathbb{R}^{n}, \mathcal{F}_{k}\right)_{0}$ and $h \in \operatorname{Diff}_{c}^{n+1}\left(\mathbb{R}^{n}, \mathcal{F}_{n-k}^{\prime}\right)_{0}$ such that $f=g \circ h$, where $\mathcal{F}_{k}=\left\{\mathbb{R}^{k} \times\{\mathrm{pt}\}\right\}$ and $\mathcal{F}_{n-k}^{\prime}=\left\{\{\mathrm{pt}\} \times \mathbb{R}^{n-k}\right\}$ are the product foliations of $\mathbb{R}^{n}$.

In fact, if $f=\left(f_{1}, f_{2}\right)$ is sufficiently close to the identity then $h=\left(h_{1}, h_{2}\right)$ given by $h(x, y)=\left(x, f_{2}(x, y)\right)$, where $x \in \mathbb{R}^{k}, y \in \mathbb{R}^{n-k}$, is a diffeomorphism which belongs to $\operatorname{Diff}_{c}^{n+1}\left(\mathbb{R}^{n}, \mathcal{F}_{n-k}^{\prime}\right)_{0}$. Define $g=\left(g_{1}, g_{2}\right)$ by $g(x, y)=\left(f_{1}\left(h^{-1}(x, y)\right), y\right)$. We have that $g \in \operatorname{Diff}_{c}^{n+1}\left(\mathbb{R}^{n}, \mathcal{F}_{k}\right)_{0}$, provided $f$ is sufficiently close to the identity. Then

$$
\begin{aligned}
(g \circ h)(x, y) & =\left(g_{1}(h(x, y)), g_{2}(h(x, y))\right)=\left(\left(f_{1} \circ h^{-1}\right)(h(x, y)), h_{2}(x, y)\right) \\
& =\left(f_{1}(x, y), f_{2}(x, y)\right)=f(x, y)
\end{aligned}
$$

is the required decomposition.

\section{References}

[1] A. Banyaga, The structure of classical diffeomorphism groups, Mathematics and its Applications 400, Kluwer Academic Publishers Group, Dordrecht, 1997.

[2] D. B. A. Epstein, The simplicity of certain groups of homeomorphisms, Compositio Math. 22 (1970), 165-173.

[3] M. R. Herman, Sur le groupe des difféomorphismes du tore, Ann. Inst. Fourier (Grenoble) 23 (1973), 75-86.

[4] J. N. Mather, Commutators of diffeomorphisms, Comment. Math. Helv. I 49 (1974), 512528; II 50 (1975), 33-40; III 60 (1985), 122-124.

[5] J. N. Mather, A curious remark concerning the geometric transfer map, Comment. Math. Helv. 59 (1984), 86-110.

[6] T. Rybicki, The identity component of the leaf preserving diffeomorphism group is perfect, Monatsh. Math. 120 (1995), 289-305.

[7] T. Rybicki, On foliated, Poisson and Hamiltonian diffeomorphisms, Diff. Geom. Appl. 15 (2001), 33-46.

[8] W. Thurston, Foliations and groups of diffeomorphisms, Bull. Amer. Math. Soc. 80 (1974), 304-307. 